\title{
UNIQUE FACTORIZATION IN MULTIPLICATIVE SYSTEMS
}

\section{R. D. JAMES AND IVAN NIVEN}

In discussing unique factorization and ideal theory, C. C. MacDuffee $[1$, p. 122$]$ cites the multiplicative system of positive integers of the form $1+7 k$ as an example where unique factorization fails, since $792=22 \cdot 36=8 \cdot 99$, and $8,22,36,99$ are all primes in the system. H. Davenport [2, p. 21] uses positive integers of the form $1+4 k$ for the same purpose, with the numerical case $693=9.77$ $=21 \cdot 33$. In this paper we examine all multiplicative systems made up of arithmetic progressions, and decide the question of unique factorization.

For a fixed positive integer $n$, let $M$ be a multiplicatively closed system of positive integers such that if $x \in M$ and $y \equiv x(\bmod n)$, $y>0$, then $y \in M$. It will be assumed that $n$ is the smallest positive integer which can be used to define $M$. For example the set $M$ of all positive integers congruent to 1,3 , or 5 modulo 6 is also the set congruent to 1 modulo 2 , and in this case $n=2$. We divide the integers $1,2, \cdots, n$ into two classes: the set $A, \phi(n)$ in number, of those relatively prime to $n$, and the others in set $B, n-\phi(n)$ in number.

TheOREM. $M$ has unique factorization if and only if $M \cap A=A$, $M \cap B=0$, i.e. if and only if $M$ consists of all positive integers relatively prime to $n$.

The proof will be in several parts, and will employ the following additional notation. Write $n=p_{1}^{\alpha_{1}} p_{2}^{\alpha_{2}} \cdots p_{r}^{\alpha_{r}}$ where the $p_{i}$ are primes of $I$, the set of all positive integers. Numbers $m$ belonging to $M$ which cannot be factored in $M$ are called pseudo-primes.

Case 1. $M \cap A=A, M \cap B=0$. Thus $m \in M$ if and only if $(m, n)=1$ and so the pseudo-primes of $M$ are the primes of $I$ with $p_{1}, p_{2}, \cdots, p_{r}$ deleted. Unique factorization in $M$ is implied by that in $I$.

Case 2. $M \cap B \neq 0, M \cap A=0$. To any $m \in M$ there corresponds $b \in(M \cap B)$ such that $m \equiv b(\bmod n)$, whence $(m, n)=(b, n)$. For any $b_{i}$ in $M \cap B$, define $d_{i}=\left(b_{i}, n\right)$, so that $d_{i}>1$. Among the elements of $M \cap B$, choose $b_{1}$ so that its corresponding $d_{1}$ is a minimum. Thus $\left(b_{1}, n\right)=d_{1}$ with say $b_{1}=d_{1} q_{1}$ and $n=d_{1} q_{2},\left(q_{1}, q_{2}\right)=1$. Choose distinct primes $\pi_{1}>n$ and $\pi_{2}>n$ of the form $q_{1}+x q_{2}$, and also the prime $\pi_{3}$ of the form $1+x n$. Since $\pi_{1}>n$ we have $\left(\pi_{1}, n\right)=1$ and $\pi_{1} \notin M$. Similarly $\pi_{2}, \pi_{3}, \pi_{1} \pi_{3}$, and $\pi_{2} \pi_{3}$ are not in $M$.

Next we establish that $d_{1} \pi_{1} \pi_{3}$, which is of the form $b_{1}+x n$, is a

Presented to the Society, May 1, 1954; received by the editors November 5, 1953. 
pseudo-prime in $M$. For any nontrivial factorization in $M$ would be either of the form $\left(\delta_{1}\right)\left(\delta_{2} \pi_{1} \pi_{3}\right)$ or $\left(\delta_{1} \pi_{1}\right)\left(\delta_{2} \pi_{3}\right)$ where $\delta_{1} \delta_{2}=d_{1}$ with $1<\delta_{1}<d_{1}$. But these are not valid factorizations in $M$, inasmuch as $\delta_{1}$ and $\delta_{1} \pi_{1}$ are not in $M$, since $\left(\delta_{1}, n\right)=\left(\delta_{1} \pi_{1}, n\right)=\delta_{1}<d_{1}$ would contradict the minimum principle used in the selection of $d_{1}$. Similarly $d_{1} \pi_{2} \pi_{3}$ is a pseudo-prime in $M$.

Also $d_{1} \pi_{1}$ and $d_{1} \pi_{2}$ are pseudo-primes in $M$. For any nontrivial factorization of $d_{1} \pi_{1}$ in $M$ would have the form $\left(\delta_{1}\right)\left(\delta_{2} \pi_{1}\right)$ with $1<\delta_{1}$ $<d_{1}$, but as before $\delta_{1}$ is not in $M$.

The proof of Case 2 is completed by observing that

$$
\left(d_{1} \pi_{1}\right)\left(d_{1} \pi_{2} \pi_{3}\right)=\left(d_{1} \pi_{2}\right)\left(d_{1} \pi_{2} \pi_{3}\right),
$$

each term in parentheses being a pseudo-prime, and the factorizations being different since $\pi_{1} \neq \pi_{2}$.

Case 3. $A \neq A \cap M \neq 0$. Let $a \in M$, so that $a^{\phi(n)} \in M$, and $a^{\phi(n)}$ $\equiv 1(\bmod n)$, so that $1 \in M$. Let $\alpha$ be a member of $A$ which is not in $M$. Since $\alpha^{\phi(n)} \in M$, there is a least exponent $e>1$ such that $\alpha^{e} \in M$. Choose distinct primes $\pi_{1}>n$ and $\pi_{2}>n$ of the form $\alpha+x n$, and it follows that $\pi_{1}^{e}$ and $\pi_{2}^{e}$ are pseudo-primes in $M$. Also $\pi_{1} \pi_{2}^{e-1}$ and $\pi_{2} \pi_{1}^{e-1}$ are pseudo-primes in $M$, so the proof is complete by the factorization

$$
\left(\pi_{1}^{e}\right)\left(\pi_{2}^{e}\right)=\left(\pi_{1} \pi_{2}^{e-1}\right)\left(\pi_{2} \pi_{1}^{e-1}\right) .
$$

Case 4. $M \cap A=A, B \neq M \cap B \neq 0$. As in Case 1 , the pseudoprimes of $M$ include all primes $p$ such that $(p, n)=1$. But since $M \cap B$ $\neq 0$, there are other pseudo-primes of $M$, and we now prove that these others have no prime factors apart from the prime factors of $n$.

If $q$ is any pseudo-prime with $(q, n)>1$, write $q=q_{1} q_{2}$ where the prime factors of $q_{1}$ are also prime factors of $n$, but $\left(q_{2}, n\right)=1$. We can readily prove that $q_{2}=1$. For the congruence $\mu q_{2} \equiv 1(\bmod n)$ has a positive solution $\mu$, and all of $\mu, q_{2}, q, \mu q$ are in $M$. Thus $\mu q \equiv q_{1}$ $(\bmod n)$, so $q_{1}$ is in $M$. Thus $q=q_{1} q_{2}$ is a factorization in $M$, and $(q, n)$ $>1$ implies $\left(q_{1}, n\right)>1$ and $q_{1} \neq 1$, so $q_{2}=1$ since $q$ is a pseudo-prime.

Thus we have established that the pseudo-primes of $M$ are of two types: (1) all primes $p$ with $(p, n)=1 ;(2)$ at least one pseudo-prime $q$ whose prime factors are contained in the set $p_{1}, p_{2}, \cdots, p_{r}$, the prime factors of $n$. Let us order these primes so that precisely $p_{1}, \cdots, p_{h}$ are the prime factors of these pseudo-primes, with $1 \leqq h \leqq r$.

LEMMA 1. $M$ lacks unique factorization if it contains more than $h$ pseudo-primes of type (2) above.

Proof. If we have $h+1$ distinct pseudo-primes 


$$
q_{j}=p_{1}^{\alpha_{1 j}} p_{2}^{\alpha_{2 j}} \cdots p_{h}^{\alpha_{h j}},
$$

$$
j=1,2, \cdots, h+1
$$

we can solve the $h$ equations

$$
\sum_{j=1}^{h+1} x_{j} \alpha_{i j}=0, \quad i=1,2, \cdots, h,
$$

for integral values $x_{j}$ not all zero. Thus we would have

$$
\prod_{j=1}^{h+1} q_{j}^{x_{j}}=1
$$

and multiplying both sides by $q_{j}^{-x_{i}}$ in all cases of negative $x_{j}$, we obtain a counter-example to unique factorization.

Lemma 2. Let $p$ be any one of the primes $p_{1}, \cdots, p_{h}$. If no pseudoprime of $M$ is of the form $p^{i}$, then $M$ has infinitely many pseudo-primes of type (2).

Proof. There is no loss of generality in taking $p=p_{1}$. Now $M$ contains a pseudo-prime with $p_{1}$ as a factor, say

$$
q=p_{1}^{\beta_{1}} p_{2}^{\beta_{2}} \cdots p_{h}^{\beta_{h}}
$$

where $\beta_{1}>0$ and $\beta_{2}+\beta_{3}+\cdots+\beta_{h}>0$ by the hypothesis of the lemma. For $j=1,2,3, \cdots$, choose positive $x_{j}$ to satisfy $x_{j}$ $\equiv p_{1}^{j}\left(\bmod n / p_{1}^{\alpha_{1}}\right)$ and $x_{j} \equiv 1\left(\bmod p_{1}\right)$. Choose a positive integer $\gamma$ so that $\gamma \beta_{1} \geqq \alpha_{1}$, so that $q^{\gamma}\left(x_{j}-p_{1}^{j}\right)$ is divisible by $n$, that is,

$$
x_{j} q^{\gamma} \equiv p_{1}^{j} q^{\gamma} \equiv p_{1}^{j+\gamma \beta_{1}} p_{2}^{\gamma \beta_{2}} \cdots p_{h}^{\gamma \beta_{h}}(\bmod n) .
$$

Also $\left(x_{j}, n\right)=1$ so that $x_{j}$ and $x_{j} q^{\gamma}$ are in $M$. If $M$ contained only a finite number of pseudo-primes of type (2), then

$$
p_{1}^{j+\gamma \beta_{1}} p_{2}^{\gamma \beta_{2}} \cdots p_{h}^{\gamma \beta_{h}}
$$

could not be factored into pseudo-primes for $j$ very large, the exponents $\gamma \beta_{2}, \cdots, \gamma \beta_{h}$ being fixed. This completes the proof of Lemma 2, and in view of Lemma 1, we can now complete Case 4 by proving the following result.

LEMMA 3. If $M$ contains only a finite number of pseudo-primes of type (2), the number exceeds $h$.

Proof. By Lemma 2, $M$ contains $h$ pseudo-primes of the form $p_{1}^{\gamma_{1}}, p_{2}^{\gamma_{2}}, \cdots, p_{h}^{\gamma_{h}}$. We assume that these are all the pseudo-primes of type (2) in $M$, and obtain a contradiction. 
First we establish that $\gamma_{1}=\gamma_{2}=\cdots=\gamma_{h}=1$. Choose the positive integer $\mu$ so that $\mu \gamma_{1} \geqq \alpha_{1}$, and the positive integer $x$ to satisfy simultaneously $x \equiv p_{1}\left(\bmod n / p_{1}^{\alpha_{1}}\right)$ and $x \equiv 1\left(\bmod p_{1}\right)$. Thus $n$ is a divisor of $p_{1}^{\mu \gamma_{1}}\left(x-p_{1}\right)$, that is

$$
x p_{1}^{\mu \gamma_{1}} \equiv p_{1}^{1+\mu \gamma_{1}}(\bmod n) \text {. }
$$

Now $(x, n)=1$, so that $x$ is in $M$, and so is $p_{1}^{\gamma_{1}}$, whence $x p_{1}^{\mu \gamma_{1}}$ is in $M$. Thus $p_{1}^{1+\mu \gamma_{1}}$ is in $M$. But by the opening remark of this proof the only powers of $p_{1}$ which are in $M$ are also powers of $p_{1}^{\gamma_{1}}$. Hence $\gamma_{1}=1$. Similarly $\gamma_{2}=\gamma_{3}=\cdots=\gamma_{h}=1$.

We have established that the pseudo-primes of type (2) in $M$ are $p_{1}, p_{2}, \cdots, p_{h}$. Thus the set $M$ can be characterized as all positive integers relatively prime to $p_{h+1}, \cdots, p_{r}$, if such primes exist. So the set $M$ can be described in terms of the modulus $p_{h+1} p_{h+2} \cdots p_{r}$, which is less than $n$ since $h \geqq 1$. This contradicts our basic hypothesis that $n$ is the smallest modulus available to define $M$. This completes the proof of Lemma 3 and Case 4.

REMARK ON THE PROOFs. The Dirichlet theorem on the infinitude of primes in an arithmetic progression is used in Cases 2 and 3, but is not essential in these proofs, as we now show.

In Case 2 it is not necessary that $\pi_{1}, \pi_{2}, \pi_{3}$ be primes, but merely that they have the following properties:

$$
\begin{aligned}
\pi_{3} & \equiv 1(\bmod n),\left(\pi_{1}, n\right)=\left(\pi_{2}, n\right)=1, \\
d_{1} \pi_{1} & \equiv d_{1} \pi_{2} \equiv b_{1}(\bmod n), \pi_{1} \neq \pi_{2} .
\end{aligned}
$$

It can be verified that these are all satisfied by the choices $\pi_{3}=1+n$, $\pi_{1}=q_{1}+u q_{2}$ where $u$ is defined as the product of all primes dividing $n$ but not dividing $q_{1}$, and $\pi_{2}=q_{1}+u p q_{2}$ where $p$ is any prime exceeding $n$.

To remove the Dirichlet theorem from the proof of Case 3 we proceed as follows. Let $p$ be the smallest integer in $A$ which is not in $M$. Our notation is justified since $p$ is a prime in $I$, for if $p=q v$ it would follow that $q$ and $v$ were in $A$ but not both in $M$, contradicting the minimal property of $p$. Define $e$ as the least exponent such that $p^{e} \in M$, and so $1<e \leqq \phi(n)$. Define $b=n+p^{e-1}$, whence $b^{e} \equiv\left(p^{e}\right)^{e-1}(\bmod n)$ so that $b \notin M$ but $b^{e} \in M$ and $p b \in M$.

Now consider the factorization in $M$, not all factors being necessarily pseudo-primes,

$$
\left(p^{e}\right)\left(b^{e}\right)=(p b)(p b) \cdots(p b) .
$$

However, $p^{e}$ is a pseudo-prime, and $p^{e}$ is not a divisor of $p b$, since $(p, n)=1$ implies $(p, b)=1$. 


\section{REFERENCES}

1. C. C. MacDuffee, Introduction to abstract algebra, Wiley, 1940.

2. H. Davenport, The higher arithmetic, Hutchinson's University Library, 1952.

University of British Columbia AND

UNIVERSITY OF OREGON

\section{ON A PROBLEM OF ADDITIVE NUMBER THEORY}

G. G. LORENTZ

Let $A, B, \cdots$ denote sets of natural numbers. The counting function $A(n)$ of $A$ is the number of elements $a \in A$ which satisfy the inequality $a \leqq n$. We shall call two sets $A, B$ complementary to each other if $A+B$ contains all sufficiently large natural numbers.

In a talk with the author $\mathrm{P}$. Erdös conjectured that each infinite set $A$ has a complementary set $B$ of asymptotic density zero. Here we wish to establish a theorem which gives an upper estimate for $B(n)$ in terms of $A(n)$. As a particular case, the truth of Erdös' conjecture will follow. The estimate (1) below should be compared with the (trivial) lower estimate $B(n) \geqq(1-\epsilon) n / A(n)$, which holds for all large $n$.

Theorem 1. For each infinite set $A$ there is a complementary set $B$ such that

$$
B(n) \leqq C \sum_{k=1}^{n} \frac{\log A(k)}{A(k)} ;
$$

$C$ is an absolute constant and the terms of the sum with $A(k)=0$ are to be replaced by one.

Proof. Let $A$ be given and let $m<n$ denote two natural numbers. We shall choose certain integers $b$ in the interval $m \leqq b<2 n$ in such a way that the sums $a+b, a \in A$, fill the whole interval $n<a+b \leqq 2 n$. Our concern will be to obtain the upper estimate (4) for the number $K$ of the $b$ 's.

First we take a $b_{1}$ in $[m, 2 n)$ in such a way that the portion of $A+b_{1}$ contained in $(n, 2 n]$ has the maximal possible number $S$ of elements and choose this $b_{1}$ as one of our $b$ 's. Then we take another

Received by the editors March 2, 1954. 